548. Ludwig Knorr: Ueber Amino-pyrazole.

[Mittheilong aus clem chem. Institut der Universităt Jena.]

(Eingegangen am 13. August 1904.)

Im Verlaufe einer umfassenden Untersuchung über das Pyrazoi, deren ansfübrliche Publication in den $\gg$ Annalen der Chemies ich soeben rorbereite, sind auch die beiden Monoaminopyrazole und ein Diaminopyrazol dargestellt worden. Ich möchte mit einem auszugsweisen Berichte über diese Basen nicht mehr lünger zögern, da inzwischen das 4-Aminopyrazol von anderer Seite ${ }^{1}$ ) durch Spaltang des Isoxanthins erhalten und in Form einiger Salze nod der Dibenzoylverbindung beschrieben worden ist. Ich beschränke mich dabei auf ganz kurze Angaben über die Darstellung der drei Basen, indem ich mir vorbehalte. die dabei anftretenden $Z$ wischenproducte a. a. O. ausführlich zu beschreiben.

\title{
I. 4-Amino-pyrazol.
}

Salze des 4-Aminopyrazols lassen sich leicht nach bekannten $\mathrm{M}_{\mathrm{e}}$ thoden aus dem Pyrazol durch das Nitropyrazol ${ }^{2}$ ) hindurch gewionen. Doch ist bei der Reduction des Nitropyrazols die Anwendung ron Salzsäure zu vermeiden, weil sonst chlorhaltige Basen entstehen, dif ron dem Amidopyrazol schwer zu trennen sind. Sehr gut verlüuft die Reduction bei Anwendung von Zinkstanb und Essigsäure. Das Ende der Reaction ist leicht an dem Verschwinden der zuerst auftretenden purpurrothen Farbe zu erkennen. Nach der Entfernung des Zinks gewinnt man beim Eindampfen mit Salzsäure das Hydrochlorat des 4-Aminopyrazols, das durch Umkrystallisiren aus Alkohol unter Verwendung von Thierkohle leicht gereinigt werden kann.

$0.1825 \mathrm{~g}$ Sbst.: $0.1522 \mathrm{~g} \mathrm{CO}_{2}, 0.0769 \mathrm{~g} \mathrm{H}_{2} \mathrm{O}$ - $0.1023 \mathrm{~g}$ Sbst.: $24.1 \mathrm{~cm} \mathrm{~N}$ $(90,731 \mathrm{~mm}) .-0.1396 \mathrm{~g}$ Sbst.: $0.2585 \mathrm{~g} \mathrm{AgCl}$ - $-0.1240 \mathrm{~g}$ Sbst.: $0.2269 \mathrm{gr}$ $\mathrm{AgCl}$.

$$
\begin{aligned}
& \mathrm{C}_{3} \mathrm{~B}_{5} \mathrm{~N}_{3} .2 \mathrm{HCl} \text {. Ber. C } 23.07, \mathrm{H} 4.49, \mathrm{~N} 26.93 \text {, Cl } 45.51 \text {. } \\
& \text { Gef. } 22.72 \text {, } 4.71 \text {, 27.23, } 45.81,45.25 .
\end{aligned}
$$

Analysirt wurden ferner das Pikrat (Schmp. 193-1940) und das Pikrolonat (Schmp. $242^{\circ}$ ) der Base, wobei die merkwürdige Beolachtung gemacht wurde, dass sich das 4-Aminopyrazol mit 2 wei Mol-külen Pikrinsäure, aber nur mit einem Molekñl Pikrolonsäure verinigt. Ferner wurden dargestellt und analysirt das Dibenzoylderirat (Schmp. 1730) und das sebr charakteristische Nitrat. Dies-

1) Behrend und Wollers, Ann. d. Chem. 323, 279 [1902].

3) Anch über diese Verbindung, die durch ihron stark sauren Charahier wiffallt und vermuthlich als Isonitroverbindung $z a$ betrachten ist, werde irts a. a. O. eingehend berichten. 
beiden Derivate der Base sind bereits von Wollers dargestellt und analysirt worden. Die Analyse des $\mathrm{Nit}$ ats sei hier mitgetheilt, weil die gefundenen Werthe ron den durch Wollers erhaltenen Zahlen abweichen; Wollers neigte anf Grund der von ihm gefundenen Werthe zur Annahme, das Salz enthalte ein halbes Molekül Krystallwasser Nach dem Ergebniss meiner Analyse ist das Salz wasserfrei.

$0.2103 \mathrm{~g}$ Sbst.: $0.1321 \mathrm{~g} \mathrm{CO}_{2}, 0.0651 \mathrm{~g} \mathrm{H}_{2} \mathrm{O} .-0.2079 \mathrm{~g}$ Sbst.: $0.1301 \mathrm{~g}$ $\mathrm{CO}_{2}, 0.0631 \mathrm{~g} \mathrm{H}_{2} \mathrm{O}$ - $0.1285 \mathrm{~g}$ Sbst.: $36.0 \mathrm{~cm} \mathrm{~N}\left(11^{0}, 757 \mathrm{~mm}\right)$.

$$
\begin{aligned}
& \mathrm{C}_{3} \mathrm{H}_{7} \mathrm{~N}_{5} \mathrm{O}_{6} \text {. Ber. C 17.23, } \quad \text { H 3.35, N } 33.49 \text {. } \\
& \text { Gef. } \gg 17.13,17.07,-3.46,3.40,33.25 \text {. }
\end{aligned}
$$

Die Darstellung der Base aus den Salzen ist Wollers nicht gelungen. Er schreibt darüber:

Bine vollstăndige Reinigung des salzsauren Salzes konnte bisher nicht erreicht werden. Ebensowenig gelang es, der freien Base habhaft zu werden: sie ist in Wasser leicht löslich und zersetzt sich, aus den Salzen durch Alkalien freigemacht, alsbald unter Braunfărbung.

In der That ist das 4-Aminopyrazol gegen den Luftsauerstoff, namentlich in alkalischer Lösung, ausserordentlich empfindlich, und seine Isolirung wird durch die Leichtlöslichkeit in Wasser und die Unlōslichkeit in Aether erschwert. Indessen liessen sich doch Methoden ausfindig machen, um diese Base in reinem Zustande zu isoliren.

Verwandelt man das Hydrochlorat der Base durch Erwärmen mit der berechneten Menge Schwefelsăure in das Sulfat, zerlegt dieses genau mit Barytlösung und dampft dann die wässrige Lösung der Base unter Durchleiten von Wasserstoffgas bei stark vermindertem Druck $a b$, so hinterbleibt die Base zunächst als rothbraunes Harz, lässt sich aber durch Sublimation im Wasserstoffstrome in prächtigen, schneeweissen Krystallen gewinnen.

Zum gleichen Ziele gelangt man, wenn man die reinen Salze der Base mit viel frisch destillirtem Essigester überschichtet und in einem mit Wasserstoff gefüllten Scheidetrichter nach Zusatz der berechneten Menge Natronlauge kräftig durchsehüttelt. Der Essigester hinterlässt die Base als rothbraune, allmählich krystallisirende Masse, aus der im Wasserstoffstrom sich ebenfalls ein schneeweisses, eisblumenähnliches Sublimat gewinnen lïsst.

$0.1241 \mathrm{~g}$ Sbst: $0.2005 \mathrm{~g} \mathrm{CO}_{2}, 0.0694 \mathrm{~g} \mathrm{H}_{2} \mathrm{O} .-0.1221 \mathrm{~g}$ Sbst.: :3.2 cem N $\left(15^{\prime \prime}, 750 \mathrm{~mm}\right)$.

$\mathrm{C}_{3} \mathrm{H}_{5} \mathrm{~N}$. Ber. C $43.37, \mathrm{H} 6.02, \mathrm{~N} 50.60$.

Gef. \44.06, \6.25, \50.37.

Die sublimirte Base schmilzt bei $80-82^{\circ}$. Sie kann in kleinen Meugen im Wasserstoffstrom bei stark vermindertem Drucke destillirt werdeu. Die Base ist hygroskopisch und löst sich spielend in Wasser. sehr schwer in Aether, Ligroïn und Benzol, reichlicher in Chloroform- 
Essigester und Alkohol. Aus der alkoholischen Löвung fällt das 4-Aminopyrazol anf Aetherzusatz zunächst amorph aus; dagegen hinterlassen die meisten Lösungsmittel beim Verüunsten die Substanz in concentrisch gruppirten Prismen. Die wässrige Lösung der Base reagirt neutral und verändert sich ebenso wie die feste Base in Berührung mit der Luft nur sehr langsam. Dagegen nimmt die Lösung bei Gegenwart von Alkali rasch erhebliche Mengen von Sauerstoff auf, wobei sie sich zuerst violettroth und mit der Zeit schwarz färbt. Die Lösungen der Salze absorbiren keinen Sauerstoff.

Ammoniakalische Silber- sowie Gold-Chloridlösung werden durch die Base schon in der Kälte krältig reducirt. Silbernitrat fallt ans der wässrigen Lösung einen weissen, Kupfersulfat einen grünen Niederschlag. Mit Eisenchlorid zeigt die Lösung der Base keine Rfaction.

Das 4-A minopyrazol lässt sich sehr leicht diazotiren. Seine Diazouiumsalze sind durch grosse Beständigkeit auggezeichnet. Sie erleiden in kochender, wässriger Lösung keine Zersetzang. Im übrigen gleichen sie den aromatischen Diazoverbindungen, lassen sich zu Azofarbstoffen kuppeln und unter gewissen Bedingungen im Sinne der bekannten Diazoreactionen umsetzen.

Durch Eintragen ron Pyrazol-4-diazoniumchloridlösung in warme Jodkaliumlösung konnte z. B. das schon von Buchner und Fritschy) beschriebene Jodpyrazol rom Schmp. $108.5^{\circ}$ gewonnen werden.

Weiteres über diese merkwürdige Base, ihre Derivate und ihre Umwandelungen wird a. a. $O$. berichtet werden.

Bei den Versuchen mit dem 4-Aminopyrazol bin ich von den HHrn. Dr. Treff und Dr. Lorentz aufs eifrigste unterstützt worden. Ich spreche den beiden Herren auch an dieser Stelle meinen besten Dank aus.

\section{3(5)-Amino-pyrazol.}

(Mitbearbeitet von H. Pemsel 2).)

Das 3(5)-A mino-pyrazol konnte aus der Pyrazol-3(5)-carbonsäure ${ }^{2}$ ) nach der Methode von Curtius durch den Aethylester (Schmp. 160 ${ }^{\circ}$ ), das Hydrazid (Schmp. 175 $)$, Azid (Zersetzungspunkt ca. $143^{\circ}$ ) der Säure und das Urethan des Aminopyrazols (Schmp. $\left.153^{\circ}\right)$ hindurch gewonnen werden. Diese $Z$ wischenproducte werde ich an anderer Stelle ausführlich beschreiben.

1) Ann. d. Chem. 273, $264 . \quad$ 2) Dissert., Jen a 1898.

3) Buchner und Papendieck, Ann. d. Chem. 273, 237 und 254: Knorr und Macdonald, Ann. d. Chem. 279, 231. 
Durch Verseifen des Urethans mit kochender Salzsänre lässt sich leicht das salzsaure 3(5)-Aminopyrazol darstellen. Die Base selbst gewinnt man am besten durch Verseifung des Urethans mit Barytlösung.

Das aus dem Azid der Pyrazol-3(5)-carbonsäure durch Kochen mit Alkohol dargestellte, aus Essigester umkrystallisirte Urethan wurde mit der dreifachen Menge Barythydrat in wäsariger Lösung einige Stunden unter Rückfluss gekocht. Schon nach kurzem Erwärmen trübte sich die Lösung unter Abscheidung von Baryumcarbonat. Nach beendeter Reaction wurde der überschüssige Baryt durch Einleiten von Kohlensäure in die kochende Lösung zur Abscheidung gebracht und das Filtrat vom Baryumcarbonat im Vacuum eingedampft. Mit den Wasserdämpfen gingen nur geringe Mengen Aminopyrazol über. Die Hauptmenge hinterblieb im Destillirkolben als dickes Oel, das durch wiederholtes Aufnehmen in absolutem Alkohol von den letzten Spuren Baryamearbonat befreit und dann durch Destillation zunächst bei Minderdruck, dann bei Atmosphärendruck gereinigt wurde.

Das 3(5)-Aminopyrazol siedet bei $282^{\circ}$ und $753 \mathrm{~mm}$ Druck, bei $218^{\circ}$ und $122 \mathrm{~mm}$ Druck. Es ist ein farbloses, dickflüssiges, hygroskopisches Oel, leicht löslich in Wasser, Alkohol and Essigester, schwer löslich in Aether.

$0.2038 \mathrm{~g}$ Sbst.: $0.3220 \mathrm{~g} \mathrm{CO}_{2}, 0.1128 \mathrm{~g} \mathrm{H}_{2} \mathrm{O}$. $-0.1240 \mathrm{~g}$ Sbst.: $54 \mathrm{ccm}$ N $\left(11^{0}, 753 \mathrm{~mm}\right)$.

$$
\begin{aligned}
& \mathrm{C}_{3} \mathrm{H}_{5} \mathrm{~N}_{3} \text {. Ber. C 43.37, } \mathrm{H} 6.02, \mathrm{~N} 50.60 \text {. } \\
& \text { Gef. » 43.09, } \$ 6.19, 》 50.26 \text {. }
\end{aligned}
$$

Die wässrige Lösung der Base reagirt neutral. Salpetersäure scheidet aus dieser Lösung kein Nitrat aus, wie dies für eine wässrige Lösung des isomeren 4-A minopyrazols charakteristisch ist. Die Base besitzt reducirende Eigenschaften. Sie reducirt Goldchlorid in der Kălte, Platinchlorid langsam in der Kälte, rasch in der Wärme. Ammoniakalische Silberlösung wird in der Wärme reducirt, in der Kälte liefert sie mit der wässrigen Lösung der Base einen weissen amorphen Njederschlag. Silbernitratlösung füllt weisse Nädelchen aus, Cblorkalklösung erzengt eine Braunfärbung, welche an die bekannte Reaction des Anilins erinnert.

Die Base unterscheidet sich son dem isomeren 4-Aminopyrazol vor allem dnrch die relativ viel grössere Beständigkeit in alkalischer Lösung. Die Lösung der Base in Natronlauge färbt sich an der Luft, ja selbst bei Durchleiten von Sauerstoff, nur schwach gelb.

Gegen salpetrige Säure verhält sich die Base ähnlich dem isomeren 4-Aminopyrazol. Salze und Derivate werden ebenfalls in der ausführlichen Mittheilung beschrieben werden. 
III. 3.5-Diamino-pyrazol.

(Mitbearbeitet von Paul Morontz). ")

Als Ausgangsmaterial zur Gewinnung des 3.5-Diamino-p y ra zols diente die Pyrazol-3.5-dicarbonsäure ${ }^{2}$ ), die nach der Methode von Curtius über das Hydrazid und Azid (Zersetzungspunkt 120$122^{\circ}$ ) der Säure leicht in das Urethan (Nadeln rom Schmp. 2220) des 3.5-Diaminopyrazols übergeführt werden konote.

Die nähere Beschreibung auch dieser $Z$ wischenproducte werde ich in der ausführlichen Abhandlung folgen lassen.

Die Verseifung des Urethans wird am besten ebenfalls mit Barythydrat bewirkt in ähnlicher Weise, wie es oben für die Darstellung des 3(5)-Aminopyrazols beschrieben worden ist.

Die vom Baryumcarbonat befreite wässrige Lösung der Base hinterliess bein Eindampfen im Vacnum einen honiggelben Syrup, der keine Neigung zum Krystallisiren zeigte, weshalb auf eine vollständige Reinigung der Base verzichtet werden musste. Immerhin zeigte eine Stickstoff bestimmung, dass die Base nach der angegebenen Methode direct in ziemlich reinem Zustand gewonnen wird.

$0.0856 \mathrm{~g}$ Sbst.: $44.4 \mathrm{ccm} \mathrm{N}\left(26^{\circ}, 750 \mathrm{~mm}\right)$.

$\mathrm{C}_{3} \mathrm{H}_{6} \mathrm{~N}_{4}$. Ber. N 57.14. Gef. N 56.6̆̌.

Die Base ist sehr leicht löslich in Wasser und Alkohol, unlöslich in Aether. Ihre wăssrige Lösung reducirt ammoniakalische Silberlösung und erzeugt in Chlorkalklösung eine Braunfärbung. Durch überschüssige salpetrige Säure wird die Base in normaler Weise diazotirt.

Das 3.5. Diaminopyrazol stimmt demnach in seinem Verbalten mit den weiter oben beschriebenen Monaminopyrazolen im Wesentlichen überein. Es gleicht mebr dem 3(5)-Aninopyrazol als dem 4-Aminopyrazol.

Von beiden unterscheidet es sich durch seine geringere Beständigkeit in saurer Lösung. Kocht man die Lösungen der Base in Säuren oder in Alkalien, so färben sich diese Lösungen tief roth. Die eingedampfte salzsaure Lösung hinterlässt einen dunkelroth gefärbten Riickstand, in welchem Chlorammonium nachgewiesen werden konnte.

Auch bei längerem Aufbewahren der Base macht sich eine allmähliche Veränderung bemerkbar. Das zunüchst helle Oel färbt sich nach und nach dunkler und wird immer zähflüssiger.

1) Dissertation, 1897.

2) Marchetti, diese Berichte 25, Ref. 744 [1892]; Buchnor und Papondieck, Ann. d. Chem. 273, 248; Knorr und Maedonald, Ann. d. Chem. $279,218$. 
Von Derivaten der Base wurden dargestellt und analysirt: der Pyrazol-3.5-dibarnstoff, das 3.5-Dibenzoyl-diaminopyrazol und das 3.j-Dibenzal-diaminopyrazol.

Von diesen Derivaten besitzt nur das Dibenzoylderivat der Base einen Schmelzpunkt. Es schmilzt unter vorhergehendem Sintern bei $207-208^{\circ}$. Diese Verbindungen werden ebenfalls später beschrieben werden; angefügt sei nur die Analyse des Harnstoffes, dureb welche die Zusammensetzung der Base als Diamino-pyrazol ausser Zweifel gestellt wird.

$0.1417 \mathrm{~g}$ Shst.: $0.1672 \mathrm{~g} \mathrm{CO}_{2}, 0.0579 \mathrm{~g} \mathrm{H}_{2} \mathrm{O} .-0.1139 \mathrm{~g}$ Sbst.: $57,5 \mathrm{cem}$ N $\left.\left(26^{\circ}, 74:\right) \mathrm{mm}\right)$.

$$
\mathrm{C}_{5} \mathrm{H}_{8} \mathrm{~N}_{6} \mathrm{O}_{2} . \quad \begin{aligned}
& \text { Ber. } \mathrm{C} 32.60, \mathrm{H} 4.34, \mathrm{~N} 45.65 . \\
& \text { Gef. 》 32.18, - 4.53, 》 45.58. }
\end{aligned}
$$

R. v. Rothenburg ${ }^{1}$ ) hat das 3.5-Diaminopyrazol wahrsebeinlich bereits in Händen gehabt. Er erbielt durch Einwirkung von Hydrazinhydrat auf Malonitril ein sdunkelbraunes Oel\&, aus dem er ein Pikrat in Form vōthlicher Blättehenc und ein Benzylidenderivat als uschlecht krystallisirenden "Niederschlag vom ungefähren Zersetzungspunkt $170^{\circ}$ herstellen konnte.

Da der für das letztere Präparat gefundene Stickstoffgehalt auf die Formel eines Dibenzal-diaminopyrazols stimmt, so dürfte jenes dunkelbraune Oel Diaminopyrazol enthalten haben. Mit Sicherheit lässt sich dies auf Grund der spärlichen Angaben von Rothenburg's nicht entscheiden.

549. James Mc Connan: Notiz über das 1-Phenyl.

\subsection{5-trimethyl-pyrazol.}

[Ans dem chemischen Institut der Universität Jena.]

(Eingegangen am 13. August 1904.)

Aus dem 1-Phenyl-3 4,4-trimethyl-5-oxypyrazolin (I) erhielten vor längerer Zeit ') Knorr und Jochheim durch Wasserentziehung eine sauerstofffreie Base, die sich mit dem aus Methylacetylaceton und Phenylhydrazin synthetisch gewonnenen 1-Phenyl-3.4.5-trimethylpyrazol (II) identisch erwies:

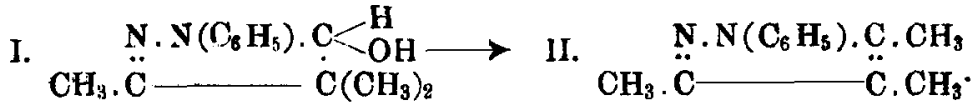

1) Diese Berichte 27, 690 [1894].

2) Inangural-Dissertation von Ernst Jochheim, Jena 1896; veróffentlicht in Cohn, Tabellarische Uebersicht der Pyrazolderivate «, 1897, S. 414 nod diese Borichte 36, 1277 [1903].

Berichte d. D. chem. Gesellschaft. Jahrg. XXXVIJ. 